\title{
Radiofrequency-Assisted Hepatic Resection
}

\author{
Madhava Pai ${ }^{1}$, Charis Kyriakides ${ }^{1}$, Sameh Mikhail ${ }^{1}$, Nagy Habib ${ }^{1}$, Duncan Spalding ${ }^{1}$, Long Jiao ${ }^{1}$, and \\ Daniel Cherqui ${ }^{2}$
}

${ }^{1}$ Hammersmith Hospital, Liver and Pancreas Surgery, Imperial College London, London, UK; ${ }^{2}$ NewYork-Presbyterian Hospital/Weill Cornell Medical Center, Surgery, New York

\begin{abstract}
Background. The only curative procedure to date for liver tumors is surgical resection, which remains a major procedure with marked morbidity and mortality. Radiofrequency (RF) has increasingly been used for both ablation and resection. On the basis of this technique, a new bipolar RF device, Habib 4X, has been developed and used clinically. We present our technique of liver resection with this device in a patient with colorectal liver metastases.

Methods. A patient with situs inversus who had colorectal liver metastases in her left lobe underwent left lateral segmentectomy with the new device, a four-electrode bipolar resection device that uses RF energy for tissue necrosis. After laparotomy and intraoperative ultrasound, the plane resection was marked $1 \mathrm{~cm}$ away from the edge
\end{abstract}

of the lesion. Coagulative desiccation was performed along this plane using this sealer connected to a RF generator. The necrosed band of parenchyma was then divided with a scalpel and resection completed.

Results. The length of the procedure was 105 minutes; resection time was 35 minutes. Total blood loss was 100 $\mathrm{ml}$. No blood transfusions were required, and the patient was not admitted to the intensive care unit after surgery. The patient was discharged 10 days after surgery without any surgical complications.

Conclusions. We think that RF-assisted liver resection with this new device is safe and effective. It is quicker than conventional RF and may reduce overall hospital stay in liver resection patients.
Electronic supplementary material The online version of this article (doi:10.1245/s10434-011-1804-2) contains supplementary material, which is available to authorized users.

(C) Society of Surgical Oncology 2011

First Received: 20 October 2010;

Published Online: 24 June 2011

N. Habib

e-mail: nagy.habib@imperial.ac.uk 